On exponentials of exponential generating series

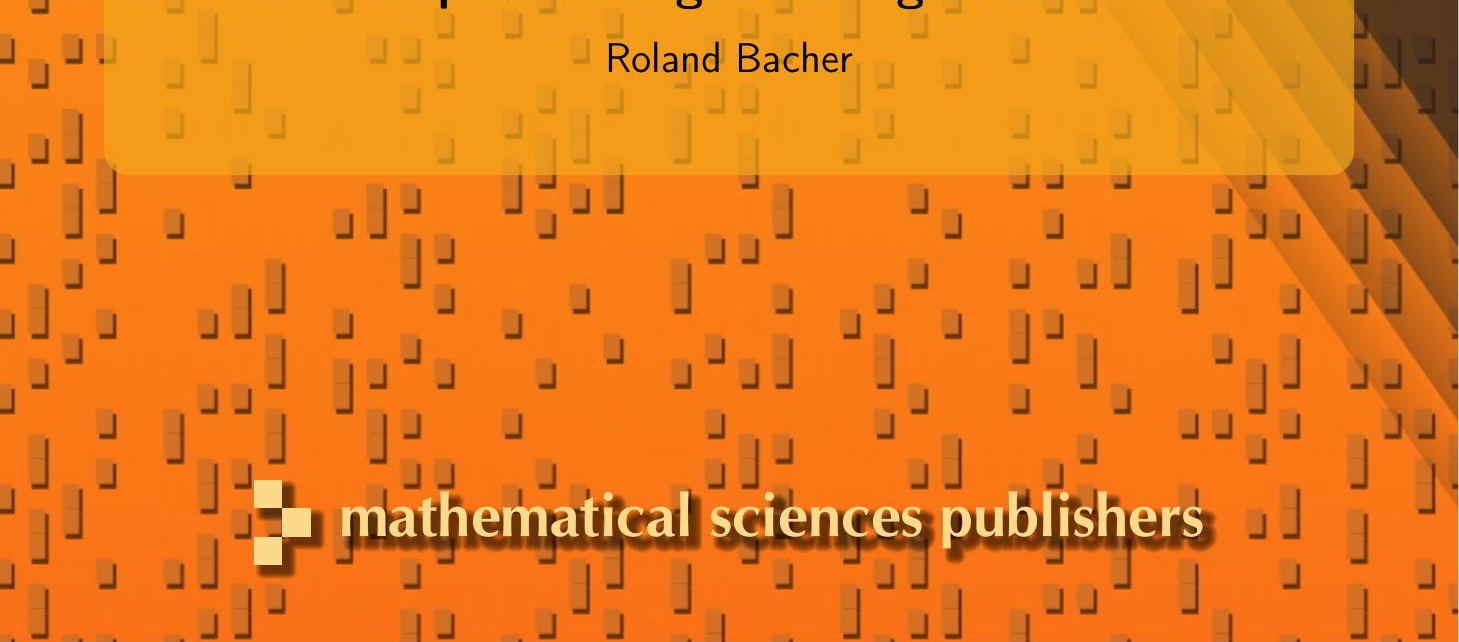

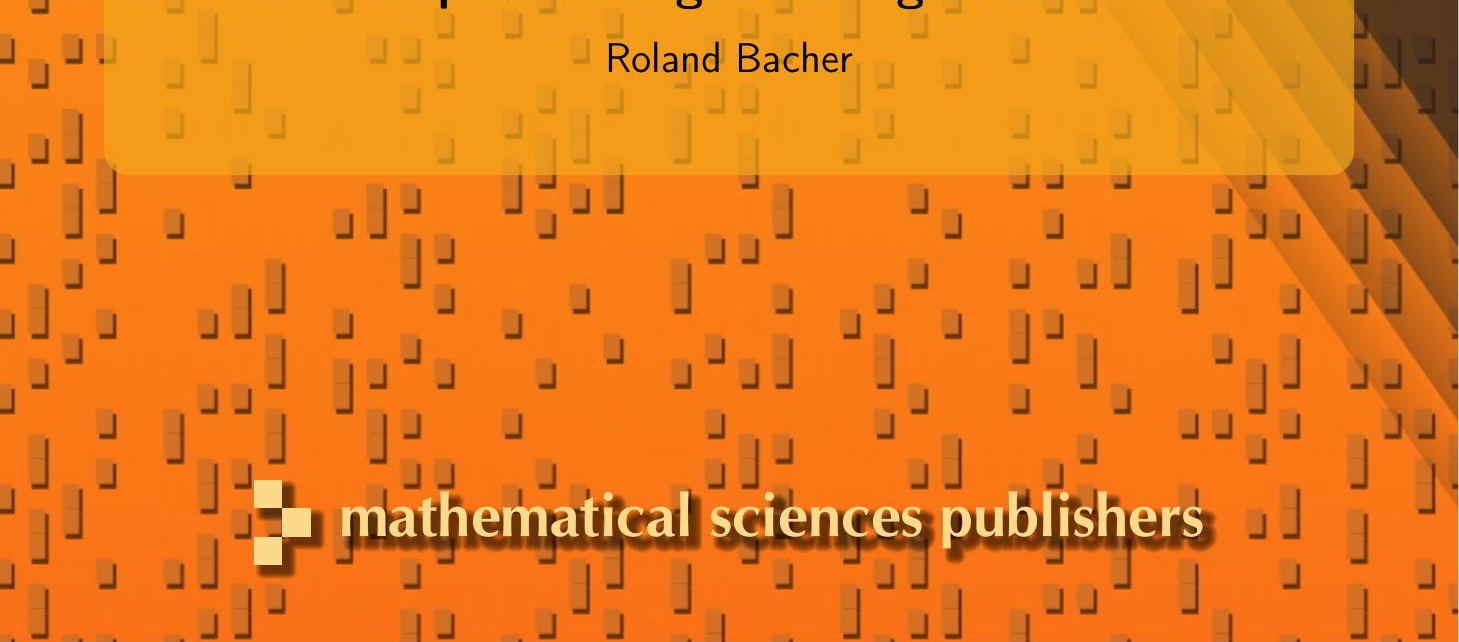

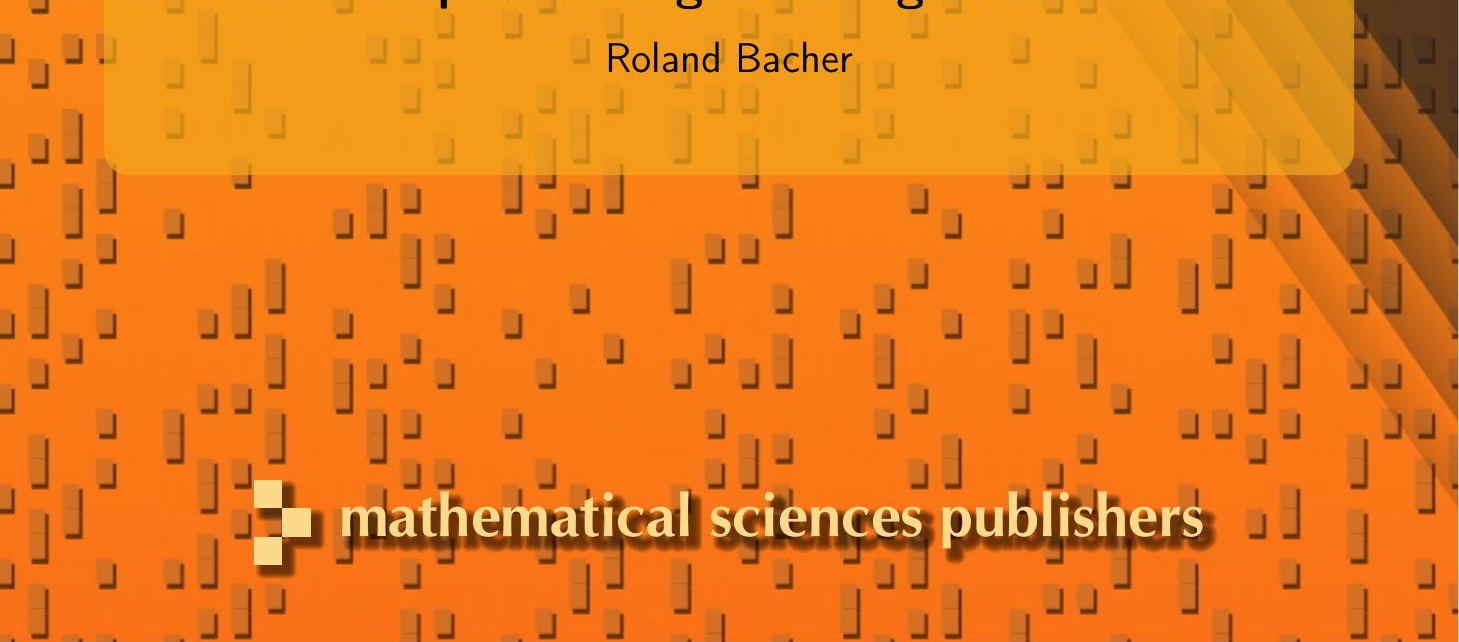

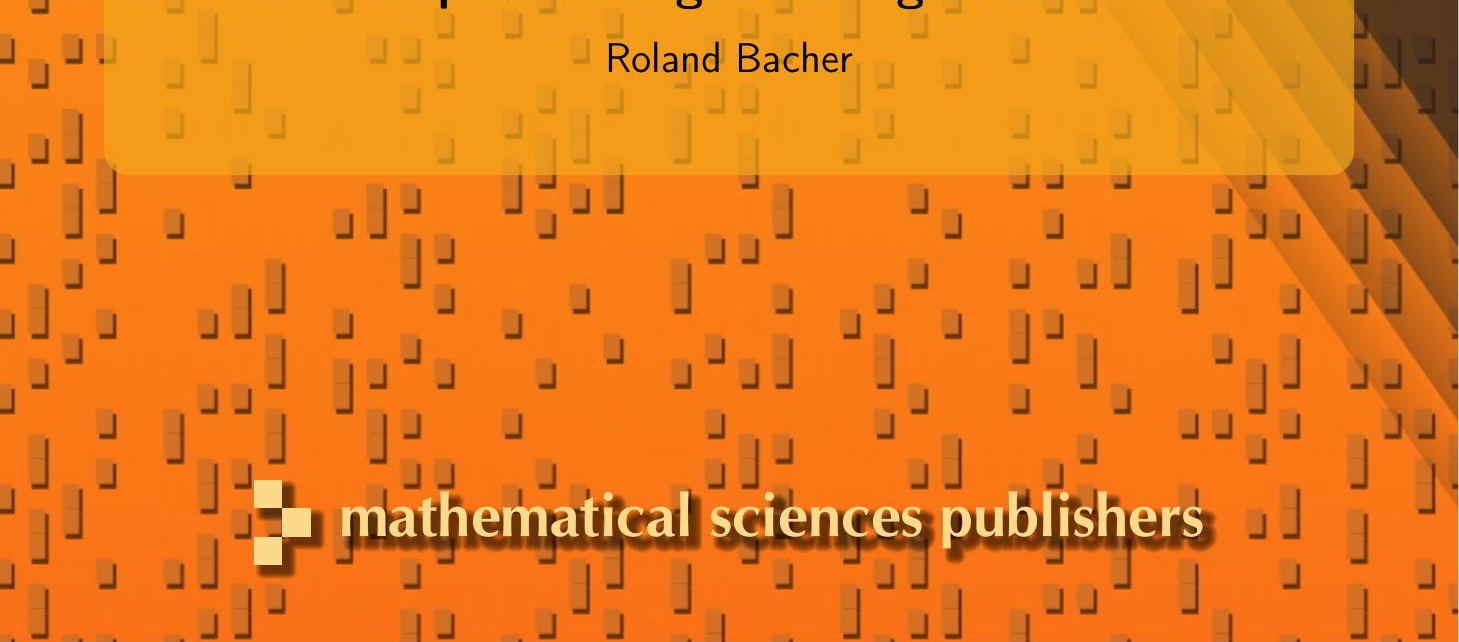

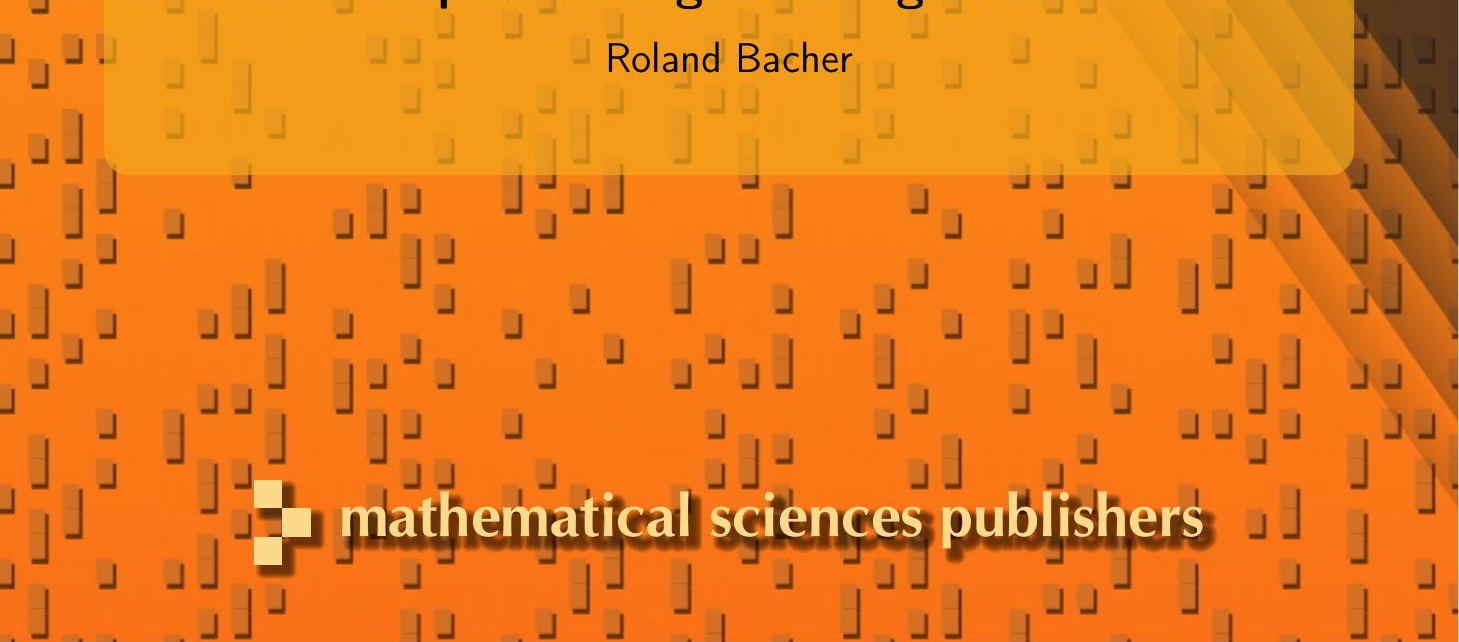




\title{
On exponentials of exponential generating series
}

\author{
Roland Bacher
}

After identification of the algebra of exponential generating series with the shuffle algebra of ordinary formal power series, the exponential map

$$
\exp _{!}: X \mathbb{K}[[X] \longrightarrow 1+X \mathbb{K} \llbracket[X]
$$

for the associated Lie group with multiplication given by the shuffle product is well-defined over an arbitrary field $\mathbb{K}$ by a result going back to Hurwitz. The main result of this paper states that exp! and its reciprocal map $\log _{\text {! }}$ induce a group isomorphism between the subgroup of rational, respectively algebraic series of the additive group $X \mathbb{R} \llbracket X \rrbracket$ and the subgroup of rational, respectively algebraic series in the group $1+X \llbracket \llbracket[X]]$ endowed with the shuffle product, if the field $\mathbb{K}$ is a subfield of the algebraically closed field $\overline{\mathbb{F}}_{p}$ of characteristic $p$.

\section{Introduction}

The equality

$$
\left(\sum_{n=0}^{\infty} \alpha_{n} \frac{X^{n}}{n !}\right)\left(\sum_{n=0}^{\infty} \beta_{n} \frac{X^{n}}{n !}\right)=\sum_{n=0}^{\infty} \sum_{m=0}^{n}\left(\begin{array}{c}
n+m \\
n
\end{array}\right) \alpha_{n} \beta_{m} \frac{X^{n+m}}{(n+m) !}
$$

shows that we can define an algebra structure on the vector space

$$
\mathscr{E}(\mathbb{K})=\left\{\sum_{n=0}^{\infty} \alpha_{n} \frac{X^{n}}{n !} \mid \alpha_{0}, \alpha_{1}, \ldots \in \mathbb{K}\right\}
$$

of formal exponential generating series with coefficients $\alpha_{0}, \alpha_{1}, \ldots$ in an arbitrary field or ring $\mathbb{K}$. For the sake of simplicity, we will work only over fields. The expression $\alpha_{n} / n$ ! should be considered formally, since the numerical value of $n$ ! is zero over a field of positive characteristic $p \leq n$.

MSC2000: primary 11B85; secondary 11B73, 11E08, 11E76, 22E65.

Keywords: Bell numbers, exponential function, shuffle product, formal power series, divided powers, rational series, algebraic series, homogeneous form, automaton sequence. 
Motivation for this work is given by the fact that the formula (1) allows us to define the shuffle product

$$
\sum_{n=0}^{\infty} \gamma_{n} X^{n}=\left(\sum_{n=0}^{\infty} \alpha_{n} X^{n}\right) \sqcup\left(\sum_{n=0}^{\infty} \beta_{n} X^{n}\right)
$$

of two formal power series $\sum_{n=0}^{\infty} \alpha_{n} X^{n}$ and $\sum_{n=0}^{\infty} \beta_{n} X^{n}$ by setting

$$
\gamma_{n}=\sum_{k=0}^{n}\left(\begin{array}{l}
n \\
k
\end{array}\right) \alpha_{k} \beta_{n-k} .
$$

The definition of the shuffle product arises in the theory of divided powers; see, for example, [Berthelot and Ogus 1978, Definition 3.1]. The main properties needed in this paper are already in [Hurwitz 1899]. I have the impression that the main results of the present paper, given by Theorems 1.1 and 1.3, do not fit very well into the theory of divided powers: they are based on an interplay between ordinary power series (used for defining rationality and algebraicity) and exponential power series (used for defining an analogue of the exponential map in positive characteristic). A special instance of this exponential map is a standard ingredient for divided powers [Berthelot and Ogus 1978, Appendix A, Proposition A1], but ordinary formal power series do not seem to play a significant role there.

Definition (2) is also a particular case of a shuffle product defined more generally for formal power series in several noncommuting variables. The associated shuffle algebras arise, for example, in the study of free Lie algebras [Reutenauer 1993], Hopf algebras and polyzetas [Zagier 1994; Cartier 2002], formal languages [Berstel and Reutenauer 1988], etc.

I became interested in this subject through the study of the properties of the algebra of recurrence matrices, a subset of sequences of matrices displaying a kind of self-similarity structure used in [Bacher 2006; 2008] for studying reductions of the Pascal triangle modulo suitable Dirichlet characters. Such recurrence matrices are closely related to automata groups and complex dynamical systems; see, for example, [Nekrashevych 2005] for details. Over a finite field, they can be identified with rational formal power series in several noncommuting variables (the underlying algebras are however very different) and it is thus natural to investigate properties of other possible products preserving these sets. The main results of this paper, Theorems 1.1 and 1.3 (and their effective analogues, Theorems 1.5 and 1.6), deal with properties of the shuffle product for formal power series in one variable that have gone unnoticed in the existing literature, as far as I am aware.

We denote by

$$
\mathfrak{m}_{\mathscr{E}}=\left\{\sum_{n=1}^{\infty} \alpha_{n} \frac{X^{n}}{n !} \mid \alpha_{1}, \alpha_{2}, \cdots \in \mathbb{K}\right\} \subset \mathscr{E}(\mathbb{K})
$$


the maximal ideal of the local algebra $\mathscr{E}(\mathbb{K})$. A straightforward computation, already known to Hurwitz [1899], shows that $a^{n} / n$ ! is always well-defined for $a \in \mathfrak{m}_{\mathscr{E}}$. Endowing $\mathbb{K}$ with the discrete topology and $\mathscr{E}(\mathbb{K})$ with the topology given by coefficientwise convergence, the functions

$$
\exp (a)=\sum_{n=0}^{\infty} \frac{a^{n}}{n !} \quad \text { and } \quad \log (1+a)=-\sum_{n=1}^{\infty} \frac{(-a)^{n}}{n}
$$

are always defined for $a \in \mathfrak{m}_{\mathscr{E}}$.

Switching back to ordinary generating series

$$
A=\sum_{n=1}^{\infty} \alpha_{n} X^{n}, \quad B=\sum_{n=1}^{\infty} \beta_{n} X^{n} \in \mathfrak{m},
$$

contained in the maximal ideal $\mathfrak{m}=X \llbracket \llbracket X \rrbracket$, of (ordinary) formal power series, we write

$$
\exp ! A=1+B
$$

if

$$
\exp \left(\sum_{n=1}^{\infty} \alpha_{n} \frac{X^{n}}{n !}\right)=1+\sum_{n=1}^{\infty} \beta_{n} \frac{X^{n}}{n !} .
$$

It is easy to see that $\exp$ ! defines a one-to-one map between $\mathfrak{m}$ and $1+\mathfrak{m}$ with reciprocal map

$$
1+B \mapsto A=\log _{!}(1+B) .
$$

It satisfies

$$
\exp _{!}(A+B)=\exp _{!} A \amalg \exp _{!} B
$$

for all $A, B \in \mathfrak{m}$, where the shuffle product

$$
\left(\sum_{n=0}^{\infty} \alpha_{n} X^{n}\right) \amalg\left(\sum_{n=0}^{\infty} \beta_{n} X^{n}\right)=\sum_{n, m=0}^{\infty}\left(\begin{array}{c}
n+m \\
n
\end{array}\right) \alpha_{n} \beta_{m} X^{n+m}
$$

corresponds to the ordinary product of the associated exponential generating series. The map exp! defines thus an isomorphism between the additive group $(\mathfrak{m},+)$ and the special shuffle group $(1+\mathfrak{m}, \amalg)$ with group-law given by the shuffle product. It coincides with the familiar exponential map from the Lie algebra $\mathfrak{m}$ into the special shuffle group, considered as an infinite-dimensional Lie group.

It follows from [Fliess 1974] that rational, respectively algebraic elements form a subgroup in $(1+\mathfrak{m}, \sqcup)$ if one works over a subfield of $\overline{\mathbb{F}}_{p}$. It is thus natural to consider the corresponding subgroups (under the reciprocal map $\log$ ! of the Lie exponential $\left.\exp _{!}: \mathfrak{m} \mapsto 1+\mathfrak{m}\right)$ in the isomorphic additive group $(\mathfrak{m},+)$ forming the Lie algebra of $(1+\mathfrak{m}, \amalg)$. The answer, which is the main result of this paper, 
is surprisingly simple: the corresponding subgroup is exactly the subgroup of all rational, respectively algebraic elements in the additive group $\mathfrak{m}$. We have thus:

Theorem 1.1. Let $\mathbb{K}$ be a subfield of the algebraically closed field $\overline{\mathbb{F}}_{p}$ of positive characteristic $p$. Given a series $A \in \mathfrak{m}=X \mathbb{K} \llbracket X \rrbracket$, the following two assertions are equivalent:

- A is rational.

- $\exp _{\text {! }}$ A is rational.

Theorem 1.1 fails in characteristic zero: the series

$$
\log _{!}(1-X)=-\sum_{n=1}^{\infty}(n-1) ! X^{n}
$$

is obviously transcendental. (This series also shows that Theorem 1.3 does not hold in characteristic zero.)

Example 1.2. The Bell numbers $B_{0}, B_{1}, B_{2}, \ldots$ (see [Comtet 1970, pp. 45-46] or [Stanley 1999, Example 5.2.4]) are the natural integers defined by

$$
\sum_{n=0}^{\infty} B_{n} \frac{x^{n}}{n !}=e^{e^{x}-1}
$$

and have combinatorial interpretations.

Since $e^{x}-1$ is the exponential generating series of the sequence $0,1,1, \ldots$, we have $\sum_{n=0}^{\infty} B_{n} x^{n}=\exp _{!}(x /(1-x))$ for the ordinary generating series

$$
\sum_{n=0}^{\infty} B_{n} x^{n}=1+x+2 x^{2}+5 x^{3}+15 x^{4}+52 x^{5}+203 x^{6}+877 x^{7}+4140 x^{8}+\cdots
$$

of the Bell numbers.

The reduction of $\sum_{n=0}^{\infty} B_{n} x^{n}$ modulo a prime $p$ is thus always a rational element of $\mathbb{F}_{p} \llbracket x \rrbracket$. A few such reductions are

$$
\frac{1}{1+x+x^{2}} \quad(\bmod 2), \frac{1+x+x^{2}}{1-x^{2}-x^{3}} \quad(\bmod 3), \frac{1+x+2 x^{2}-x^{4}}{1-x^{4}-x^{5}} \quad(\bmod 5) .
$$

Theorem 1.3. Let $\mathbb{K}$ be a subfield of the algebraically closed field $\overline{\mathbb{F}}_{p}$ of positive characteristic $p$. Given a series $A \in \mathfrak{m}=X \mathbb{\ll} \llbracket X \rrbracket$, the following two assertions are equivalent:

- A is algebraic.

- $\exp _{!}$A is algebraic.

Theorems 1.1 and 1.3 are the main results of this paper and can be restated as follows. 
Corollary 1.4. Over a subfield $\mathbb{\subset} \subset \overline{\mathbb{F}}_{p}$, the group isomorphism

$$
\exp _{!}:(\mathfrak{m},+) \longrightarrow(1+\mathfrak{m}, \sqcup)
$$

restricts to an isomorphism between the subgroups of rational elements in $(\mathfrak{m},+)$ and in $(1+\mathfrak{m}, \amalg)$.

It restricts also to an isomorphism between the subgroups of algebraic elements in $(\mathfrak{m},+)$ and in $(1+\mathfrak{m}, \sqcup)$.

In particular, the subgroup of rational, respectively algebraic elements in the shuffle group $(1+\mathfrak{m}, \sqcup)$ is a Lie-group whose Lie algebra (over $\mathbb{R} \subset \overline{\mathbb{F}}_{p}$ ) is given by the additive subgroup of all rational, respectively algebraic elements in $(\mathfrak{m},+)$.

Theorems 1.1 and 1.3 can be made more precise as follows.

Given a rational series $A \in \mathbb{K}[[X]]$ represented by a reduced fraction $f / g$, where $f, g$ with $g \neq 0$ are two coprime polynomials of degrees $\operatorname{deg} f$ and $\operatorname{deg} g$, we set $\|A\|=\max (1+\operatorname{deg} f, \operatorname{deg} g)$; see also Proposition 2.1 for a well-known equivalent description of $\|A\|$.

Theorem 1.5. We have

$$
\left\|\exp _{!} A\right\| \leq p^{q\|A\|} \quad \text { and } \quad\left\|\log _{!}(1+A)\right\| \leq 1+\|1+A\|^{p}
$$

for a rational series $A$ in $\left.\mathfrak{m} \subset \overline{\mathbb{F}}_{p} \llbracket X\right]$ having all its coefficients in a finite subfield $\mathbb{F}_{q} \subset \overline{\mathbb{F}}_{p}$ containing $q=p^{e}$ elements.

The bounds for $\log _{\text {! }}$ (and the analogous bounds in the algebraic case) can be improved; see Proposition 7.1.

Theorem 1.5 could be called an effective version of Theorem 1.1: given a rational series represented by $f / g \in \mathfrak{m}$ with $f, g \in \overline{\mathbb{F}}_{p}[X]$, Theorem 1.1 ensures the existence of polynomials $u, v$ such that $\exp _{!}(f / g)=u / v$. Theorem 1.5 shows that $u$ and $v$ are of degree at most $p^{q\|f / g\|}$. They can thus be recovered as suitable Padé approximants from the series development of $\exp$ ! $(f / g)$ up to order $2 p^{q^{\|f / g\|}}$. Experimentally, the number $\| \exp$ ! $A \|$ is generally much smaller.

Since the bounds for $\log$ ! are better than for exp!, the determination of the rational series $B=\exp _{\text {! }} A$ with $A \in \mathfrak{m}$ rational is best done as follows: start by "guessing" the rational series $B$ and check (or improve the guess for $B$ in case of failure) that $A=\log _{\text {! }}(B)$ using the bounds for $\log _{\text {! }}$.

Given a prime $p$ and a formal power series $C=\sum_{n=0}^{\infty} \gamma_{n} X^{n}$ in $\mathbb{K} \llbracket X \rrbracket$ with coefficients in a subfield $\mathbb{K}$ of $\overline{\mathbb{F}}_{p}$, we define for $f \in \mathbb{N}, k \in \mathbb{N}, k<p^{f}$ the series

$$
C_{k, f}=\sum_{n=0}^{\infty} \gamma_{k+n p^{f}} X^{n} .
$$


The vector space $\mathscr{K}(C)=\mathbb{} C+\sum_{k, f} \mathbb{K} C_{k, f}$ spanned by $C$ and by all series of the form $C_{k, f}, k \in\left\{0, \ldots, p^{f}-1\right\}, f \in\{1,2, \ldots\}$ is called the $p$-kernel of $C$. We denote its dimension by $\kappa(C)=\operatorname{dim} \mathscr{K}(C)$.

Algebraic series in $\mathbb{K}\left[[X]\right.$ for $\mathbb{K}$ a subfield of $\overline{\mathbb{F}}_{p}$ are characterized by a theorem of Christol [Allouche and Shallit 2003, Theorem 12.2.5] stating that a series $C$ in $\overline{\mathbb{F}}_{p} \llbracket X \rrbracket$ is algebraic if and only if its $p$-kernel $\mathscr{K}(C)$ is of finite dimension $\kappa(C)<$ $\infty$. We have $\kappa(A+B) \leq \kappa(A)+\kappa(B)$, and an algebraic series $A \in \overline{\mathbb{F}}_{p} \llbracket X \rrbracket$ has a minimal polynomial of degree at most $p^{\kappa(A)}$ with respect to $A$.

Theorem 1.6. We have

$$
\kappa\left(\exp _{!} A\right) \leq q^{\kappa(A)-1} p^{q^{\kappa(A)}} \quad \text { and } \quad \kappa\left(\log _{!}(1+A)\right) \leq 1+4(\kappa(1+A))^{p}
$$

for a nonzero algebraic series $A$ in $\mathfrak{m} \subset \overline{\mathbb{F}}_{p}[[X]]$ having all its coefficients in a finite subfield $\mathbb{F}_{q} \subset \overline{\mathbb{F}}_{p}$ containing $q=p^{e}$ elements.

Considerations similar to those made after Theorem 1.5 are valid and Theorem 1.6 can be turned into an algorithmically effective version of Theorem 1.3.

A map $\mu: \mathscr{V} \longrightarrow \mathscr{W}$ between two $\mathbb{K}$-vector spaces is a homogeneous form of degree $d$ if $l \circ \mu: \mathscr{V} \longrightarrow \mathbb{K}$ is homogeneous of degree $d$ (given by a homogeneous polynomial of degree $d$ with respect to coordinates) for all linear forms $l: \mathcal{W} \longrightarrow \mathbb{K}$.

A useful ingredient for proving Theorems 1.1, 1.3 and their effective versions is the following characterization of $\log$ ! :

Proposition 1.7. Over a field $\mathbb{K} \subset \overline{\mathbb{F}}_{p}$, the application $\log _{\text {! }}: 1+\mathfrak{m} \longrightarrow \mathfrak{m}$ extends to a homogeneous form of degree $p$ from $\mathbb{K}[[X]$ into $\mathfrak{m}$.

Example 1.8. In characteristic 2, we have

$$
\log _{!}\left(\sum_{n=0}^{\infty} \alpha_{n} X^{n}\right)=\sum_{n=0}^{\infty} \alpha_{2^{n}}^{2} X^{2^{n+1}}+\sum_{0 \leq i<j}\left(\begin{array}{c}
i+j \\
i
\end{array}\right) \alpha_{i} \alpha_{j} X^{i+j}
$$

for $\sum_{n=0}^{\infty} \alpha_{n} X^{n}$ in $1+X \overline{\mathbb{F}}_{2} \llbracket X \rrbracket$.

Remark 1.9. Defining $f_{\text {! }}$ as

$$
f_{!}\left(\sum_{n=1}^{\infty} \alpha_{n} X^{n}\right)=\sum_{n=1}^{\infty} \beta_{n} X^{n}
$$

if

$$
f\left(\sum_{n=1}^{\infty} \alpha_{n} \frac{X^{n}}{n !}\right)=\sum_{n=1}^{\infty} \beta_{n} \frac{X^{n}}{n !},
$$

Theorems 1.1, 1.3, 1.5 and 1.6 have analogues for the functions sin! and tan! (and for their reciprocal functions $\arcsin _{!}$and $\arctan _{!}$). 
The rest of the paper is organized as follows. In Sections 2-6, we recall a few definitions and well-known facts that are essentially standard knowledge in the theory of divided powers; see [Berthelot and Ogus 1978] or the original work [Roby 1963; 1965]. Section 7 contains the proofs for all results mentioned above.

In a second part, starting at Section 8, we generalize Theorems 1.1 and 1.5 to formal power series in several noncommuting variables.

\section{Rational and algebraic elements in $\mathbb{K} \llbracket X \rrbracket$}

This section recalls a few well-known facts concerning rational and algebraic elements in the algebra $\mathbb{K}[[X]]$ of formal power series.

We denote by $\tau: \mathbb{K}[[X] \longrightarrow \mathbb{K} \llbracket X \rrbracket$ the shift operator

$$
\tau\left(\sum_{n=0}^{\infty} \alpha_{n} X^{n}\right)=\sum_{n=0}^{\infty} \alpha_{n+1} X^{n}
$$

acting on formal power series. The following well-known result characterizes rational series:

Proposition 2.1. A formal power series $A=\sum_{n=0}^{\infty} \alpha_{n} X^{n}$ of $\mathbb{K}[[X]$ is rational if and only if the series $A, \tau(A), \tau^{2}(A), \ldots, \tau^{k}(A)=\sum_{n=0}^{\infty} \alpha_{n+k} X^{n}, \ldots$ span $a$ finite-dimensional vector space in $\mathbb{K} \llbracket X \rrbracket$.

More precisely, the vector space spanned by $A, \tau(A), \tau^{2}(A), \ldots, \tau^{i}(A), \ldots$ has dimension $\|A\|=\max (1+\operatorname{deg} f, \operatorname{deg} g)$ if $f / g$, with $f, g \in \mathbb{K}[X]$, is a reduced expression of a rational series $A$.

The function $A \mapsto\|A\|$ satisfies the inequality

$$
\|A+B\| \leq\|A\|+\|B\|
$$

for rational series $A, B$ in $\mathbb{K}[[X]]$. As a particular case, we have

$$
\|A\|-1 \leq\|1+A\| \leq\|A\|+1 .
$$

Given a prime $p$ and a formal power series $C=\sum_{n=0}^{\infty} \gamma_{n} X^{n}$ in $\overline{\mathbb{F}}_{p} \llbracket X \rrbracket$, we denote by $\kappa(C) \in \mathbb{N} \cup\{\infty\}$ the dimension of its $p$-kernel

$$
\mathscr{K}(C)=\mathbb{K} C+\sum_{f, k} \overline{\mathbb{F}}_{p} C_{k, f},
$$

spanned by $C$ and all series of the form

$$
C_{k, f}=\sum_{n=0}^{\infty} \gamma_{k+n p^{f}} X^{n},
$$

with $k \in \mathbb{N}$ such that $k<p^{f}$ for $f \in\{1,2, \ldots\}$. 
Algebraic series of $\mathbb{K}[[X]]$, for $\mathbb{K}$ a subfield of the algebraic closure $\overline{\mathbb{F}}_{p}$ of finite prime characteristic $p$, are characterized by the following theorem of Christol [1979] (see also [Allouche and Shallit 2003, Theorem 12.2.5]):

Theorem 2.2. A formal power series $C=\sum_{n=0}^{\infty} \gamma_{n} X^{n}$ of $\overline{\mathbb{F}}_{p} \llbracket X \rrbracket$ is algebraic if and only if the dimension $\kappa(C)=\operatorname{dim} \mathscr{K}(C)$ of its p-kernel $\mathscr{K}(C)$ is finite.

Finiteness of $\kappa(C)$ amounts to recognizability of $C$, which has the following well-known consequence.

Corollary 2.3. An algebraic series of $\overline{\mathbb{F}}_{p}[[X]]$ has all its coefficients in a finite subfield of $\overline{\mathbb{F}}_{p}$.

Proposition 2.4. Let $C=\sum_{n=0}^{\infty} \gamma_{n} X^{n}$ be an algebraic series with coefficients in a subfield $\mathbb{K} \subset \overline{\mathbb{F}}_{p}$.

(i) $\mathscr{K}(\tau(C)) \subset \mathscr{K}(C)+\tau(\mathscr{K}(C))$, which implies $\kappa(\tau(C)) \leq 2 \kappa(C)$.

(ii) $\mathscr{K}(C) \subset \mathbb{K}+\mathscr{K}(\tau(C))+X \mathscr{K}(\tau(C))$, which implies $\kappa(C) \leq 1+2 \kappa(\tau(C))$.

Proof. Assertion (i) follows from an iterated application of the easy computations

$$
(\tau(C))_{k, 1}=C_{k+1,1},
$$

if $0 \leq k<p-1$ and

$$
(\tau(C))_{p-1,1}=\tau\left(C_{0,1}\right) .
$$

The proof of assertion (ii) is similar.

\section{The shuffle algebra}

This section recalls mostly well-known results concerning shuffle products of elements in the set $\mathbb{K} \llbracket X \rrbracket$ of formal power series over a commutative field $\mathbb{K}$, which is arbitrary unless specified otherwise.

The shuffle product

$$
A \amalg B=C=\sum_{n=0}^{\infty} \gamma_{n} X^{n}
$$

of $A=\sum_{n=0}^{\infty} \alpha_{n} X^{n}$ and $B=\sum_{n=0}^{\infty} \beta_{n} X^{n}$ is defined by

$$
\gamma_{n}=\sum_{k=0}^{n}\left(\begin{array}{l}
n \\
k
\end{array}\right) \alpha_{k} \beta_{n-k},
$$

and corresponds to the usual product $a b=c$ of the associated exponential generating series

$$
a=\sum_{n=0}^{\infty} \alpha_{n} \frac{X^{n}}{n !}, \quad b=\sum_{n=0}^{\infty} \beta_{n} \frac{X^{n}}{n !}, \quad c=\sum_{n=0}^{\infty} \gamma_{n} \frac{X^{n}}{n !} .
$$


The shuffle algebra is the algebra $(\mathbb{K}[[X]], \Psi)$ obtained by endowing the vector space $\mathbb{K}[[X]$ of ordinary generating series with the shuffle product. By construction, the shuffle algebra is isomorphic to the algebra $\mathscr{E}(\mathbb{K})$ of exponential generating series. In characteristic zero, the trivial identity

$$
\sum_{n=0}^{\infty} \alpha_{n} X^{n}=\sum_{n=0}^{\infty}\left(n ! \alpha_{n}\right) \frac{X^{n}}{n !}
$$

gives an isomorphism between the usual algebra $\mathbb{K} \llbracket X \rrbracket$ of ordinary generating series and the shuffle algebra $(\mathbb{K} \llbracket X \rrbracket, \sqcup)$.

The identity

$$
\left(\sum_{n \geq 0} \lambda^{n} X^{n}\right) \amalg\left(\sum_{n \geq 0} \mu^{n} X^{n}\right)=\sum_{n \geq 0}(\lambda+\mu)^{n} X^{n},
$$

equivalent to $e^{\lambda X} e^{\mu X}=e^{(\lambda+\mu) X}$, implies that the convergence radius of the shuffle product of two complex series with strictly positive convergence radii $\rho_{1}, \rho_{2}$ is at least the harmonic mean $1 /\left(1 / \rho_{1}+1 / \rho_{2}\right)$ of $\rho_{1}$ and $\rho_{2}$.

Proposition 3.1. The shift operator

$$
\tau\left(\sum_{n=0}^{\infty} \alpha_{n} X^{n}\right)=\sum_{n=0}^{\infty} \alpha_{n+1} X^{n}
$$

acts as a derivation on the shuffle algebra.

Proof. The map $\tau$ is clearly linear. The computation

$$
\begin{aligned}
\tau\left(\sum_{i, j \geq 0}\left(\begin{array}{c}
i+j \\
i
\end{array}\right) \alpha_{i} \beta_{j} X^{i+j}\right) & =\sum_{\substack{i, j \geq 0 \\
i+j \geq 1}}\left(\begin{array}{c}
i+j \\
i
\end{array}\right) \alpha_{i} \beta_{j} X^{i+j-1} \\
& =\sum_{\substack{i, j \geq 0 \\
i+j \geq 1}}\left(\left(\begin{array}{c}
i+j-1 \\
i-1
\end{array}\right)+\left(\begin{array}{c}
i+j-1 \\
j-1
\end{array}\right)\right) \alpha_{i} \beta_{j} X^{i+j-1}
\end{aligned}
$$

shows that $\tau$ satisfies the Leibniz rule $\tau(A \amalg B)=\tau(A) \amalg B+A \amalg \tau(B)$.

Proposition 3.1 is trivial and well-known in characteristic zero: the usual derivation $d / d X$ acts obviously as the shift operator on the algebra $\mathscr{E}(\mathbb{K})$ of exponential generating series over a field of characteristic zero.

The following two results seem to be due to Fliess [1974, Proposition 6].

Proposition 3.2. Shuffle products of rational power series are rational.

More precisely, we have

$$
\|A \sqcup B\| \leq\|A\|\|B\|,
$$

for two rational series $A, B$ in $\mathbb{K} \llbracket X \rrbracket$. 
Proof. Proposition 3.1 implies $\tau^{n}(A \sqcup B)=\sum_{k=0}^{n}\left(\begin{array}{l}n \\ k\end{array}\right) \tau^{k}(A) \amalg \tau^{n-k}(B)$. The series $\tau^{n}(A \amalg B)$ belongs thus to the vector space spanned by shuffle products with factors in the vector spaces $\sum_{n \geq 0} \mathbb{K} \tau^{n}(A)$ and $\sum_{n \geq 0} \mathbb{K} \tau^{n}(B)$. This implies the inequality. Proposition 2.1 ends the proof.

Proposition 3.3. Shuffle products of algebraic series in $\overline{\mathbb{F}}_{p}[[X]]$ are algebraic.

More precisely, we have

$$
\kappa(A \sqcup B) \leq \kappa(A) \kappa(B) .
$$

Proof. Denoting by $C_{k, f}$ the series

$$
C_{k, f}=\sum_{n=0}^{\infty} \gamma_{k+n p^{f}} X^{n}
$$

associated to a series $C=\sum_{n=0}^{\infty} \gamma_{n} X^{n}$, as in Section 2, and by $\kappa(C)$ the dimension of the vector space $\mathscr{K}(C)=\mathbb{K} C+\sum_{k, f} \overline{\mathbb{F}}_{p} C_{k, f}$, Lucas's identity [1878]

$$
\left(\begin{array}{l}
n \\
k
\end{array}\right) \equiv \prod_{i \geq 0}\left(\begin{array}{c}
\nu_{i} \\
\kappa_{i}
\end{array}\right) \quad(\bmod p)
$$

for $n=\sum_{i \geq 0} v_{i} p^{i}$ and $k=\sum_{i \geq 0} \kappa_{i} p^{i}$ with $v_{i}, \kappa_{i} \in\{0, \ldots, p-1\}$, implies

$$
(A \amalg B)_{k, 1}=\sum_{i=0}^{k}\left(\begin{array}{c}
k \\
i
\end{array}\right) A_{i, 1} \amalg B_{k-i, 1},
$$

for $k=0, \ldots, p-1$. Iteration of this formula shows that $(A \amalg B)_{k, f}$ (for arbitrary $k, f \in \mathbb{N}$ such that $k<p^{f}$ ) belongs to the vector space spanned by shuffle products with factors in the vector spaces $\mathscr{K}(A)$ and $\mathscr{K}(B)$ of dimension $\kappa(A)$ and $\kappa(B)$.

Christol's Theorem (Theorem 2.2) ends the proof.

Remark 3.4. Given a subfield $\mathbb{K}$ of $\overline{\mathbb{F}}_{p}$, let $\mathscr{A} \subset \mathbb{K}[[X]$ denote a vector space of finite dimension $a=\operatorname{dim} \mathscr{A}$ containing the $p$-kernel $\mathscr{K}(A)$ of every element $A \in \mathscr{A}$.

We consider an element $B=A_{1} \sqcup A_{2} \sqcup \cdots \sqcup A_{k}$ given by the shuffle product of $k$ series $A_{1}, \ldots, A_{k} \in \mathcal{A}$. Expressing all elements $A_{1}, A_{2}, \ldots$ as linear combinations of elements in a fixed basis of $\mathscr{A}$ and using commutativity of the shuffle product, the proof of Proposition 3.3 shows that the inequality $\kappa(B) \leq$ $\kappa\left(A_{1}\right) \kappa\left(A_{2}\right) \cdots \leq a^{k}=(\operatorname{dim} \mathscr{A})^{k}$ can be improved to

$$
\kappa(B) \leq\left(\begin{array}{c}
k+a-1 \\
a-1
\end{array}\right),
$$

where the binomial coefficient gives the dimension of the vector space of homogeneous polynomials of degree $k$ in $a$ (commuting) variables $X_{1}, X_{2}, \ldots, X_{a}$. 


\section{The special shuffle group}

We call the group of units of the shuffle algebra ( $\mathbb{K} \llbracket X \rrbracket, \sqcup)$ the shuffle group. Its elements are given by the set $\mathbb{K}^{*}+X \mathbb{K}[[X]]$ underlying the multiplicative unit group. The shuffle group is the direct product of the unit group $\mathbb{K}^{*}$ of $\mathbb{K}$ with the special shuffle group $(1+X \mathbb{K} \llbracket X \rrbracket, \sqcup)$.

The inverse in the shuffle group of $1-A \in(1+X \mathbb{K} \llbracket X \rrbracket, \sqcup)$ is given by

$$
\sum_{n=0}^{\infty} A^{\amalg^{n}}=1+A+A \amalg A+A \amalg A \amalg A+\cdots,
$$

where $A^{\uplus^{0}}=1$ and $A^{山^{n+1}}=A \sqcup A^{山^{n}}$ for $n \geq 1$.

The trivial identity $X \sqcup X^{n}=\left(\begin{array}{c}n+1 \\ 1\end{array}\right) X^{n+1}=(n+1) X^{n+1} \in \mathbb{K}[[X]]$ implies $(1-X) \sqcup\left(\sum_{n=0}^{\infty} n ! X^{n}\right)=1$. Invertible rational (analytical) power series have thus generally a transcendental (nonanalytical) shuffle inverse over the complex numbers.

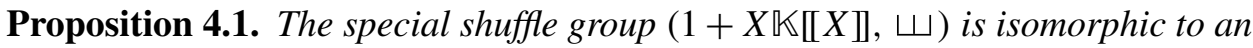
infinite-dimensional $\mathbb{F}_{p}$-vector space if the field $\mathbb{K}$ is of positive characteristic $p$.

Proposition 4.1 shows that $(1+X \mathbb{R} \llbracket X \rrbracket, \sqcup)$ is not isomorphic to the multiplicative group structure on $1+X \mathbb{R}[\llbracket X]$ if $\mathbb{K}$ is of positive characteristic.

Proof of Proposition 4.1. It follows from the fact that exp! is a group isomorphism between the $\mathbb{F}_{p}$-vector space $\mathfrak{m}$ and the special shuffle group.

Proposition 4.1 follows also as a special case from Proposition 8.1. This yields a different proof, which is not based on properties of exp!

Remark 4.2. One can show that a rational fraction $A \in 1+X \mathbb{C}[[X]]$ has a rational inverse for the shuffle product if and only if $A=1 /(1-\lambda X)$ with $\lambda \in \mathbb{C}$. (Compute $A \amalg B=1$ using the decomposition into simple fractions of the rational series $A, B$.)

\section{The exponential and the logarithm for exponential generating functions}

Hurwitz showed that $1 /(k !) a^{k}$ is well-defined for $a \in \mathfrak{m}_{\mathscr{E}}$ with coefficients in an arbitrary field or commutative ring [Hurwitz 1899, Satz 1]. We give a different proof of this fact, that implies that exp! and $\log _{\text {! }}$ are well-defined over fields of positive characteristic.

Proposition 5.1. For all natural numbers $j, k \geq 1$, the set $\{1, \ldots, j k\}$ can be partitioned in exactly

$$
\frac{(j k) !}{(j !)^{k} k !}
$$


different ways into $k$ unordered disjoint subsets of $j$ elements. In particular, the rational number in (3) is an integer for all natural numbers $j, k$ such that $j \geq 1$.

Proof. The multinomial coefficient $(j k) ! /(j !)^{k}$ counts the number of ways of partitioning $\{1, \ldots, j k\}$ into an ordered sequence of $k$ disjoint subsets containing all $j$ elements. Dividing by $k$ ! removes the order on these $k$ subsets.

This proves that the formula defines an integer for all $j, k \geq 1$, and integrality obviously also holds for $k=0$ and $j \geq 1$.

Remark 5.2. A slightly different proof of Proposition 5.1 follows from the observation that $(j k) ! /\left((j !)^{k} k !\right)$ is the index in the symmetric group over $j k$ elements of the subgroup formed by all permutations stabilizing a partition of the set $\{1, \ldots, j k\}$ into $k$ disjoint subsets of $j$ elements.

A different proof is given by the formula

$$
\frac{(j k) !}{(j !)^{k} k !}=\prod_{n=1}^{k}\left(\begin{array}{c}
n j-1 \\
j-1
\end{array}\right)
$$

easily shown using induction on $k$; see [Berthelot and Ogus 1978, Section 3] (which contains a small misprint).

Proposition 5.3. For any natural integer $k \in \mathbb{N}$, there exist polynomials $P_{k, n} \in$ $\mathbb{N}\left[\alpha_{1}, \ldots, \alpha_{n}\right]$ such that

$$
\frac{1}{k !}\left(\sum_{n=1}^{\infty} \alpha_{n} \frac{X^{n}}{n !}\right)^{k}=\sum_{n=0}^{\infty} P_{k, n}\left(\alpha_{1}, \alpha_{2}, \ldots, \alpha_{n}\right) \frac{X^{n}}{n !} .
$$

Proof. The contribution of a monomial

$$
\alpha_{1}^{j_{1}} \alpha_{2}^{j_{2}} \ldots \alpha_{s}^{j_{s}} \frac{X^{\sum_{i=1}^{s} i j_{i}}}{\left(\sum_{i=1}^{s} i j_{i}\right) !}
$$

with $j_{1}+j_{2}+\cdots+j_{s}=k$, to $\frac{1}{k !}\left(\sum_{n=1}^{\infty} \alpha_{n} X^{n} / n !\right)^{k}$ is given by

$$
\frac{1}{k !} \frac{k !}{\left(j_{1}\right) !\left(j_{2}\right) ! \ldots\left(j_{s}\right) !} \frac{\left(\sum_{i=1}^{s} i j_{i}\right) !}{\prod_{i=1}^{s}(i !)^{j_{i}}}=\left(\prod_{i=1}^{s} \frac{\left(i j_{i}\right) !}{(i !)^{j_{i}}\left(j_{i}\right) !}\right) \frac{\left(\sum_{i=1}^{s} i j_{i}\right) !}{\prod_{i=1}^{s}\left(i j_{i}\right) !},
$$

where the last expression is a product of a natural integer by Proposition 5.1 and of a multinomial coefficient. It is thus a natural integer.

Corollary 5.4. For $a=\sum_{n=1}^{\infty} \alpha_{n} X^{n} / n !$, the formulae

$$
\exp \left(\sum_{n=1}^{\infty} \alpha_{n} \frac{X^{n}}{n !}\right)=\sum_{k=0}^{\infty} \sum_{n=0}^{\infty} P_{k, n}\left(\alpha_{1}, \ldots, \alpha_{n}\right) \frac{X^{n}}{n !}
$$


and

$$
\log \left(1+\sum_{n=1}^{\infty} \alpha_{n} \frac{X^{n}}{n !}\right)=\sum_{k=1}^{\infty} \sum_{n=0}^{\infty}(-1)^{k+1}(k-1) ! P_{k, n}\left(\alpha_{1}, \ldots, \alpha_{n}\right) \frac{X^{n}}{n !}
$$

define the exponential function and the logarithm of an exponential generating series in $a \in \mathfrak{m}_{\mathscr{E}}$ and $1+a \in 1+\mathfrak{m}_{\mathscr{E}}$, respectively, over an arbitrary field $\mathbb{K}$. These functions are one-to-one and mutually reciprocal.

The following result shows that the functions exp! and $\log _{\text {! behave as expected }}$ under the derivation $\tau: \sum_{n=0}^{\infty} \alpha_{n} X^{n} \mapsto \sum_{n=0}^{\infty} \alpha_{n+1} X^{n}$ of the shuffle algebra.

Proposition 5.5. For all $A \in \mathfrak{m}=X \mathbb{K} \llbracket X \rrbracket$ over an arbitrary field $\mathbb{K}$, we have

$$
\tau\left(\exp _{!} A\right)=\left(\exp _{!} A\right) \amalg \tau(A)
$$

and

$$
\tau\left(\log _{!}(1+A)\right)=(1+A)^{\uplus^{-1}} \amalg \tau(A),
$$

where $(1+A)^{-1}$ denotes the shuffle inverse of $(1+A)$.

Proof. Proposition 3.1 implies the formal identities

$$
\tau\left(\sum_{n=0}^{\infty} \frac{A^{\varpi^{n}}}{n !}\right)=\sum_{n=0}^{\infty} n \frac{A^{\uplus^{n-1}}}{n !} \sqcup \tau(A)=\left(\sum_{n=0}^{\infty} \frac{A^{\uplus^{n}}}{n !}\right) \sqcup \tau(A),
$$

for $A \in \mathfrak{m}$. By Proposition 5.1, this identity holds over the ring $\mathbb{Z}$ and thus over an arbitrary commutative field. This establishes the formula for exp!.

For $\log _{\text {! }}$ we get similarly

$$
\tau\left(-\sum_{n=1}^{\infty} \frac{(-A)^{\sqcup^{n}}}{n}\right)=\sum_{n=1}^{\infty} n \frac{(-A)^{\uplus^{n-1}}}{n} \sqcup \tau(A)=\left(\sum_{n=0}^{\infty}(-A)^{\uplus^{n}}\right) \sqcup \tau(A),
$$

which implies the result, by Proposition 5.1 and by the trivial identity

$$
(1+A)^{\sqcup^{-1}}=\sum_{n=0}^{\infty}(-A)^{\uplus^{n}},
$$

for the shuffle inverse $(1+A)^{\Psi^{-1}}$ of $1+A \in 1+\mathfrak{m}$.

\section{The logarithm as a $p$-homogeneous form over $\overline{\mathbb{F}}_{p} \llbracket x \rrbracket$}

Given a fixed prime number $p$, Proposition 4.1 implies that there exist polynomials $Q_{p, n} \in \mathbb{N}\left[\alpha_{0}, \ldots, \alpha_{n}\right]$ for $n \geq 1$ such that

$$
\left(\sum_{n=0}^{\infty} \alpha_{n} X^{n}\right)^{\uplus^{p}}=\alpha_{0}^{p}+p \sum_{n=1}^{\infty} Q_{p, n}\left(\alpha_{0}, \ldots, \alpha_{n}\right) X^{n} .
$$


The polynomials $Q_{p, n}$ are homogeneous of degree $p$ with respect to the variables $\alpha_{0}, \ldots, \alpha_{n}$, and we denote by

$$
\mu_{p}\left(\sum_{n=0}^{\infty} \alpha_{n} X^{n}\right)=\sum_{n=1}^{\infty} Q_{p, n}\left(\alpha_{0}, \ldots, \alpha_{n}\right) X^{n}
$$

the $p$-homogeneous form defined by the ordinary generating series of the polynomials $Q_{p, 1}, Q_{p, 2}, \ldots$.

Proposition 6.1. The restriction of $\mu_{p}$ to $1+\mathfrak{m} \subset \overline{\mathbb{F}}_{p}[[X]]$ coincides with the function $\log$ !.

Proof. We have

$$
\tau\left(\mu_{p}(1+A)\right)=(1+A)^{\sqcup^{p-1}} \amalg \tau(1+A)
$$

for $A$ in $\mathfrak{m}$, where $\tau\left(\sum_{n=0}^{\infty} \alpha_{n} X^{n}\right)=\sum_{n=0}^{\infty} \alpha_{n+1} X^{n}$ is the shift operator of Proposition 3.1. This identity defines the restriction of the $p$-homogeneous form $\mu_{p}$ to $1+\mathfrak{m}$. Proposition 5.5 and the identity

$$
(1+A)^{\amalg^{p-1}} \amalg(1+A)=1
$$

show that the function $\log$ ! satisfies the same equation

$$
\tau\left(\log _{!}(1+A)\right)=(1+A)^{\sqcup^{p-1}} \amalg \tau(1+A) .
$$

Since both series $\mu_{p}(1+A)$ and $\log _{!}(1+A)$ are without constant term, the equality $\tau\left(\mu_{p}(1+A)\right)=\tau\left(\log _{!}(1+A)\right)$ implies $\mu_{p}(1+A)=\log _{!}(1+A)$.

\section{Proofs}

Proposition 7.1. If $A$ in $\left.X \overline{\mathbb{F}}_{p}[X]\right]$ is rational (respectively algebraic), then the formal power series $\log _{!}(1+A)$ is rational (respectively algebraic).

More precisely,

$$
\left\|\log _{!}(1+A)\right\| \leq 1+\left(\begin{array}{c}
p+\|1+A\|-1 \\
p
\end{array}\right) \leq 1+\|1+A\|^{p}
$$

for A rational in $\mathfrak{m}=X \overline{\mathbb{F}}_{p} \llbracket X \rrbracket$, and

$$
\kappa\left(\log _{!}(1+A)\right) \leq 1+4 \kappa(A)\left(\begin{array}{c}
p+\kappa(1+A)-2 \\
p-1
\end{array}\right) \leq 1+4(\kappa(1+A))^{p}
$$

for A algebraic in $\mathfrak{m}$.

Proposition 7.2. If $A$ in $X \overline{\mathbb{F}}_{p}\left[[X]\right.$ is rational (respectively algebraic), then $\exp _{!} A$ is rational (respectively algebraic).

More precisely, denoting by $q=p^{e}$ the cardinality of a finite field $\mathbb{F}_{q} \subset \overline{\mathbb{F}}_{p}$ containing all coefficients of $A$,

$$
\|\exp ! A\| \leq p^{q\|A\|}
$$


for A rational in $\mathfrak{m}$, and

$$
\kappa(\exp ! A) \leq q^{\kappa(A)-1} p^{q^{\kappa(A)}}
$$

for A algebraic and nonzero in $\mathfrak{m}$.

Theorems 1.1, 1.3, 1.5 and 1.6 are now simple reformulations of Propositions 7.1 and 7.2 .

Proof of Proposition 7.1. Apply the identity $(1+A)^{\amalg^{p}}=1$, which follows from Proposition 4.1, to

$$
\tau\left(\log _{!}(1+A)\right)=(1+A)^{\uplus^{-1}} \amalg \tau(A)
$$

of Proposition 5.5, to establish

$$
\tau\left(\log _{!}(1+A)\right)=(1+A)^{\uplus^{p-1}} \sqcup \tau(A),
$$

already encountered in the proof of Proposition 6.1. This shows

$$
\| \tau\left(\log _{!}(1+A)\|\leq\| 1+A\left\|^{p-1}\right\| \tau(A)\|\leq\| 1+A \|^{p}\right.
$$

and implies

$$
\left\|\log _{!}(1+A)\right\| \leq 1+\|1+A\|^{p} .
$$

This proves the cruder inequality in the rational case. The finer inequality follows from the fact that all $p$ factors of

$$
(1+A)^{\uplus^{p-1}} \sqcup \tau(A)=\tau\left(\log _{!}(1+A)\right)
$$

belong to a common vector space of dimension $\|1+A\|$ that is closed for the shift map. The details are the same as for Remark 3.4.

For algebraic $A$ we have similarly

$$
\begin{aligned}
\kappa\left(\tau\left(\log _{!}(1+A)\right)\right) & \leq(\kappa(1+A))^{p-1} \kappa(\tau(A))=(\kappa(1+A))^{p-1} \kappa(\tau(1+A)) \\
& \leq(\kappa(1+A))^{p-1} 2 \kappa(1+A) \leq 2(\kappa(1+A))^{p},
\end{aligned}
$$

using Proposition 2.4(i). This shows

$$
\kappa\left(\log _{!}(1+A)\right) \leq 1+2 \kappa\left(\tau\left(\log _{!}(1+A)\right)\right) \leq 1+4(\kappa(1+A))^{p},
$$

by Proposition 2.4(ii), and ends the proof for the cruder inequality.

The finer inequality follows from Proposition 2.4 and Remark 3.4.

Given a vector space $\mathscr{V} \subset \mathbb{K} \llbracket X \rrbracket$ containing $\mathbb{K}$, we denote by $\Gamma(\mathscr{V})$ the shuffle subgroup generated by all elements of $\mathscr{V} \cap(1+X \mathbb{R}[X]])$.

Lemma 7.3. Every element of a vector space $\mathscr{V} \subset \mathbb{K} \llbracket X \rrbracket$ containing the field $\mathbb{K}$ of constants can be written as a linear combination of elements in $\Gamma(\mathcal{V})$. 
Proof. We have the identity

$$
A=(1-\epsilon(A)+A)+(\epsilon(A)-1),
$$

where $\epsilon\left(\sum_{n=0}^{\infty} \alpha_{n} X^{n}\right)=\alpha_{0}$ is the augmentation map and where $(1-\epsilon(A)+A)$ and the constant $(\epsilon(A)-1)$ are both in $\mathbb{K} \Gamma(\mathscr{V})$ for $A \in \mathscr{V}$.

Proof of Proposition 7.2 for A rational. Corollary 2.3 shows that we can work over a finite subfield $\mathbb{K}=\mathbb{F}_{q}$ of $\overline{\mathbb{F}}_{p}$ consisting of $q=p^{e}$ elements.

Given a rational series $A$ in $\mathfrak{m}=X \mathbb{K} \llbracket X \rrbracket$, we denote by $\Gamma_{A}$ the shuffle subgroup generated by all elements of the set

$$
\left\{\bigcup_{n=0}^{\infty}\left(\tau^{n}(A)+\mathbb{K}\right)\right\} \cap\{1+X \mathbb{K} \llbracket X \rrbracket\} .
$$

This generating set of $\Gamma_{A}$ contains at most $q^{\|A\|}$ elements. Proposition 4.1 implies thus that $\Gamma_{A}$ is a finite group having at most $p^{q^{\|A\|}}$ elements. The subalgebra $\mathbb{K}\left[\Gamma_{A}\right] \subset \mathbb{K}\left[[X]\right.$ spanned by all elements of $\Gamma_{A}$ is thus of dimension $\leq p^{q^{\|A\|}}$. The identity

$$
\tau(\exp ! A)=\exp _{!} A \amalg \tau(A)
$$

of Proposition 5.5 and the fact that the derivation $\tau$ of $\mathbb{K} \llbracket X \rrbracket$ restricts to a derivation of the subalgebra $\mathbb{K}\left[\Gamma_{A}\right]$ show the inclusion

$$
\tau^{n}\left(\exp _{!} A\right) \in \exp _{!} A \sqcup \mathbb{K}\left[\Gamma_{A}\right]
$$

for all $n \in \mathbb{N}$ by Lemma 7.3. This ends the proof, since the right side is a $\mathbb{K}$-vector space of dimension at most $p^{q^{\|A\|}}$.

Proposition 7.4. We have, for every prime number $p$ and for all natural integers $j, k$ such that $j \geq 1$, the identity

$$
\frac{(j k) !}{(j !)^{k} k !} \equiv \frac{(p j k) !}{((p j) !)^{k} k !} \quad(\bmod p) .
$$

Proof. The fraction on the right side yields the cardinality of the set $\mathscr{E}$ of all partitions of $\{1, \ldots, p j k\}$ into $k$ subsets of $p j$ elements. Consider the group $G$ generated by the $j k$ cycles of length $p$ of the form $(i, i+j k, i+2 j k, \ldots, i+(p-1) j k)$ for $i=1, \ldots, j k$. The group $G$ has $p^{j k}$ elements and acts on the set of partitions by preserving their type defined as the multiset of cardinalities of all involved parts. In particular, it acts by permutation on the set $\mathscr{E}$. A partition $P \in \mathscr{E}$ is a fixpoint for $G$ if and only if every part of $P$ is a union of $G$-orbits. Choosing a bijection between $\{1, \ldots, j k\}$ and $G$-orbits of $\{1, \ldots, p j k\}$, fixpoints of $\mathscr{E}$ are in bijection with partitions of the set $\{1, \ldots, j k\}$ into $k$ subsets of $j$ elements. The number of fixpoints of the $G$-action on $\mathscr{E}$ equals thus $(j k) ! /\left((j !)^{k} k !\right)$. Since $G$ is a $p$-group, 
the cardinalities of all nontrivial $G$-orbits of $\mathscr{E}$ are strictly positive powers of $p$. This ends the proof.

Corollary 7.5. exp! and $\log$ ! commute with the "Frobenius substitution"

$$
\varphi\left(\sum_{n=0}^{\infty} \alpha_{n} X^{n}\right)=\sum_{n=0}^{\infty} \alpha_{n} X^{p n}
$$

for series in $X \overline{\mathbb{F}}_{p} \llbracket X \rrbracket$ and $1+X \overline{\mathbb{F}}_{p}[\lceil]$, respectively.

This implies $\left(\exp _{!} A\right)_{0, f}=\exp _{!} A_{0, f}$, where $C_{k, f}=\sum_{n=0}^{\infty} \gamma_{k+n p^{f}} X^{n}$ for $C=$ $\sum_{n=0}^{\infty} \gamma_{n} X^{n}$.

Lemma 7.6. $(B \sqcup C)_{0,1}=B_{0,1} \sqcup C_{0,1}$.

Proof. Follows from the identity $\left(\begin{array}{c}p n \\ k\end{array}\right) \equiv 0(\bmod p)$ if $k \neq \equiv 0(\bmod p)$.

Proof of Proposition 7.2 for A algebraic. We work again over a finite subfield $\mathbb{K}=\mathbb{F}_{q} \subset \overline{\mathbb{F}}_{p}$ containing all coefficients of $A$. Let $\Gamma_{A}$ denote the shuffle subgroup generated by all elements in

$$
(\mathscr{K}(A)+\mathbb{K}) \cap(1+X \mathbb{K} \llbracket X \rrbracket),
$$

where

$$
\mathscr{K}(A)=\mathbb{K} A+\sum_{k, f} \mathbb{K} A_{k, f}
$$

denotes the $p$-kernel of $A$. We denote by $\left.\left.\mathbb{K}\left[\Gamma_{A}\right] \subset(\mathbb{K} \llbracket X]\right], \sqcup\right)$ the shuffle subalgebra of dimension at most $p^{q^{\kappa(A)}}$ spanned by all elements of the group $\Gamma_{A} \subset$ $(1+X \mathbb{K} \llbracket X], \sqcup)$.

Using the convention $A_{0,0}=A$, we have, for $B \in \mathbb{K}[\Gamma(A)]$ and for $k$ such that $0 \leq k<p$,

$$
\begin{aligned}
\left(\exp _{!}\left(A_{0, f}\right) \amalg B\right)_{k, 1} & =\left(\tau^{k}\left(\exp _{!}\left(A_{0, f}\right) \sqcup B\right)\right)_{0,1} \\
& =\left(\sum_{j=0}^{k}\left(\begin{array}{c}
k \\
j
\end{array}\right) \tau^{j}\left(\exp _{!} A_{0, f}\right) \amalg \tau^{k-j}(B)\right)_{0,1} \\
& =\sum_{j=0}^{k}\left(\begin{array}{c}
k \\
j
\end{array}\right)\left(\tau^{j}\left(\exp _{!} A_{0, f}\right)\right)_{0,1} \amalg B_{k-j, 1},
\end{aligned}
$$

where the last equality is due to Lemma 7.6 (and to the equality $\left(\tau^{k}(C)\right)_{0,1}=C_{k, 1}$ for $0 \leq k<p$ ).

Proposition 5.5 gives $\tau\left(\exp _{!} A_{0, f}\right)=\left(\exp _{!} A_{0, f}\right) \amalg \tau\left(A_{0, f}\right)$; iterating this identity shows that $\tau^{j}\left(\exp _{!} A_{0, f}\right)$ is of the form $\left(\exp _{!} A_{0, f}\right) \amalg F$, where $F$ is 
a linear combination of shuffle products involving at most $j$ factors of the set $\left\{\tau\left(A_{0, f}\right), \tau^{2}\left(A_{0, f}\right), \ldots, \tau^{j}\left(A_{0, f}\right)\right\}$. Applying Lemma 7.6, we get

$$
\left(\tau^{j}\left(\exp _{!} A_{0, f}\right)\right)_{0,1}=\left(\exp _{0, f+1}(A)\right) \sqcup F_{0,1} .
$$

An iterated application of Lemma 7.6 now shows that $F_{0,1}$ is a linear combination of shuffle products involving at most $j$ factors in $\left\{A_{1, f+1}, \ldots, A_{j, f+1}\right\}$. We thus have $F_{0,1} \in \mathbb{K}\left[\Gamma_{A}\right]$, by Lemma 7.3 , and we get the inclusion

$$
\left(\exp _{!}\left(A_{0, f}\right) \sqcup \mathbb{K}\left[\Gamma_{A}\right]\right)_{k, 1} \subset \exp _{!}\left(A_{0, f+1}\right) \amalg \mathbb{K}\left[\Gamma_{A}\right]
$$

for all $f \in \mathbb{N}$ and for all $k \in\{0, \ldots, p-1\}$.

Setting

$$
E_{A}=\left\{\exp _{!} B \mid B \in \mathscr{K}(A) \cap X \llbracket \llbracket X \rrbracket\right\},
$$

we have the inclusion

$$
\mathscr{K}\left(\exp _{!} A\right) \subset E_{A} \uplus \mathbb{K}\left[\Gamma_{A}\right] \subset \mathbb{K}\left[E_{A}\right] \sqcup \mathbb{K}\left[\Gamma_{A}\right],
$$

where $\mathscr{K}\left(\exp _{!} A\right)$ denotes the $p$-kernel of $\exp _{\text {! }} A$. This implies

$$
\kappa(\exp ! A) \leq \operatorname{dim} \mathbb{K}\left[E_{A}\right] \operatorname{dim} \mathbb{K}\left[\Gamma_{A}\right] .
$$

We suppose now $A$ that is nonzero. The vector space $\mathscr{K}(A) \cap X \llbracket \llbracket[X]]$ is thus of codimension 1 in $\mathscr{K}(A)$. The image $E_{A}$ of $\left.\mathscr{K}(A) \cap X \mathbb{K} \llbracket X\right]$ under the group isomorphism exp! : $(X \mathbb{K}[[X]],+) \mapsto(1+X \mathbb{K}[[X]], \sqcup)$ is hence a subgroup of cardinality $q^{\kappa(A)-1}$ in $(1+X \mathbb{K}[[X]$, $\amalg)$. We have thus

$$
\kappa\left(\exp _{!} A\right) \leq \operatorname{dim} \mathbb{K}\left[E_{A}\right] \operatorname{dim} \mathbb{K}\left[\Gamma_{A}\right] \leq q^{\kappa(A)-1} p^{q^{\kappa(A)}},
$$

which ends the proof.

\section{Power series in free noncommuting variables}

This and the next section recall a few basic and well-known facts concerning (rational) power series in free noncommuting variables; see, for instance, [Stanley 1999] or [Berstel and Reutenauer 1988]. Sometimes, however, we use a different terminology, motivated by [Bacher 2008].

We denote by $\mathscr{L}^{*}$ the free monoid on a finite set $\mathscr{X}=\left\{X_{1}, \ldots, X_{k}\right\}$. We write 1 for the identity element and we use a boldface capital $\boldsymbol{X}$ for a noncommutative monomial $\boldsymbol{X}=X_{i_{1}} X_{i_{2}} \ldots X_{i_{l}} \in \mathscr{X}^{*}$. We denote by

$$
A=\sum_{\boldsymbol{X} \in \mathscr{L}^{*}}(A, \boldsymbol{X}) \boldsymbol{X} \in \mathbb{K}\left\langle\left\langle X_{1}, \ldots, X_{k}\right\rangle\right\rangle
$$

a noncommutative formal power series, where $\mathscr{Q}^{*} \ni \boldsymbol{X} \mapsto(A, X) \in \mathbb{K}$ stands for the coefficient function. 
We denote by $\mathfrak{m} \subset \mathbb{K}\left\langle\left\langle X_{1}, \ldots, X_{k}\right\rangle\right\rangle$ the maximal ideal consisting of formal power series without constant coefficient, and by $\mathbb{K}^{*}+\mathfrak{m}=\mathbb{K}\left\langle\left\langle X_{1}, \ldots, X_{k}\right\rangle\right\rangle \backslash \mathfrak{m}$ the unit group of the algebra $\mathbb{K}\left\langle\left\langle X_{1}, \ldots, X_{k}\right\rangle\right\rangle$ consisting of all (multiplicatively) invertible elements in $\mathbb{K}\left\langle\left\langle X_{1}, \ldots, X_{k}\right\rangle\right\rangle$. The unit group is isomorphic to the direct product $\mathbb{K}^{*} \times(1+\mathfrak{m})$, where $\mathbb{K}^{*}$ is the central subgroup consisting of nonzero constants and where $1+\mathfrak{m}$ denotes the multiplicative subgroup given by the affine subspace formed by power series with constant coefficient 1 . We have $(1-A)^{-1}=$ $1+\sum_{n=1}^{\infty} A^{n}$ for the multiplicative inverse $(1-A)^{-1}$ of an element $1-A \in 1+\mathfrak{m}$.

The shuffle algebra. The shuffle product $\boldsymbol{X} \sqcup \boldsymbol{X}^{\prime}$ of two noncommutative monomials $\boldsymbol{X}, \boldsymbol{X}^{\prime} \in \mathscr{X}^{*}$ of degrees $a=\operatorname{deg} \boldsymbol{X}$ and $b=\operatorname{deg} \boldsymbol{X}^{\prime}$ (for the obvious grading given by $\left.\operatorname{deg} X_{1}=\cdots=\operatorname{deg} X_{k}=1\right)$ is the sum of all $\left(\begin{array}{c}a+b \\ a\end{array}\right)$ monomials of degree $a+b$ obtained by shuffling in all possible ways the linear factors (elements of $\mathscr{X}$ ) involved in $\boldsymbol{X}$ with the linear factors of $\boldsymbol{X}^{\prime}$. A monomial involved in $\boldsymbol{X} \amalg \boldsymbol{X}^{\prime}$ can be thought of as a monomial of degree $a+b$ whose linear factors are colored by two colors with $\boldsymbol{X}$ corresponding to the product of all linear factors of the first color and $\boldsymbol{X}^{\prime}$ corresponding to the product of the remaining linear factors. The shuffle product $\boldsymbol{X} \sqcup \boldsymbol{X}^{\prime}$ can also be recursively defined by $\boldsymbol{X} \sqcup 1=1 \sqcup \boldsymbol{X}=\boldsymbol{X}$ and

$$
\left(\boldsymbol{X} X_{s}\right) \amalg\left(\boldsymbol{X}^{\prime} X_{t}\right)=\left(\boldsymbol{X} \sqcup\left(\boldsymbol{X}^{\prime} X_{t}\right)\right) X_{s}+\left(\left(\boldsymbol{X} X_{s}\right) \amalg \boldsymbol{X}^{\prime}\right) X_{t},
$$

where $X_{s}, X_{t} \in \mathscr{X}=\left\{X_{1}, \ldots, X_{k}\right\}$ are monomials of degree 1 .

Extending the shuffle product in the obvious way to formal power series endows the vector space $\mathbb{K}\left\langle\left\langle X_{1}, \ldots, X_{k}\right\rangle\right\rangle$ with an associative and commutative algebra structure called the shuffle algebra. In the case of one variable $X=X_{1}$, we recover the definition of Section 3.

The group $\mathrm{GL}_{k}(\mathbb{K})$ acts on the vector space $\mathbb{K}\left\langle\left\langle X_{1}, \ldots, X_{k}\right\rangle\right\rangle$ by linear substitutions. This action induces an automorphism of the multiplicative (noncommutative) algebra-structure or of the (commutative) shuffle algebra-structure underlying the vector space $\mathbb{K}\left\langle\left\langle X_{1}, \ldots, X_{k}\right\rangle\right\rangle$.

Substitution of all variables $X_{j}$ of formal power series in $\mathbb{K}\left\langle\left\langle X_{1}, \ldots, X_{k}\right\rangle\right\rangle$ by $X$ (or more generally by arbitrary not necessarily equal formal power series without constant term) yields a homomorphism of (shuffle) algebras into the commutative (shuffle) algebra $\mathbb{K} \llbracket X \rrbracket$.

The commutative unit group (set of invertible elements for the shuffle product) of the shuffle algebra, given by the set $\mathbb{K}^{*}+\mathfrak{m}$, is isomorphic to the direct product $\mathbb{K}^{*} \times(1+\mathfrak{m})$, where $1+\mathfrak{m}$ is endowed with the shuffle product. The inverse of an element $1-A \in(1+\mathfrak{m}, \sqcup)$ is given by

$$
\sum_{n=0}^{\infty} A^{\varpi^{n}}=1+A+A \amalg A+A \amalg A \amalg A+\cdots .
$$


The following result generalizes Proposition 4.1:

Proposition 8.1. Over a field of positive characteristic $p$, the subgroup $1+\mathfrak{m}$ of the shuffle group is an infinite-dimensional $\mathbb{F}_{p}$-vector space.

Proof. Contributions to a $p$-fold shuffle product $A_{1} \amalg A_{2} \amalg \cdots \sqcup A_{p}$ are given by monomials with linear factors colored by $p$ colors $\{1, \ldots, p\}$ keeping track of their "origin" with coefficients given by the product of the corresponding "monochromatic" coefficients in $A_{1}, \ldots, A_{p}$. A permutation of the colors $\{1, \ldots, p\}$ (and in particular, a cyclic permutation of all colors) leaves such a contribution invariant if $A_{1}=\cdots=A_{p}$. Coefficients of strictly positive degree in $A^{\uplus^{p}}$ are thus zero in characteristic $p$.

As in the one-variable case, one can prove that

$$
\frac{1}{k !} A^{\uplus^{k}}
$$

is defined over an arbitrary field $\mathbb{K}$ for $A \in \mathfrak{m}$. Monomials contributing to $A^{山^{k}}$ can be considered as colored by $k$ colors and the $k$ ! possible color-permutations yield identical contributions.

For $A \in \mathfrak{m}$, we denote by

$$
\exp ! A=\sum_{n=0}^{\infty} \frac{1}{n !} A \uplus^{n}
$$

the resulting exponential map from the Lie algebra $\mathfrak{m}$ into the infinite-dimensional commutative Lie group $(1+\mathfrak{m}, \sqcup)$. As expected, its reciprocal function is

$$
\log _{!}(1+A)=\sum_{n=1}^{\infty} \frac{(-1)^{n+1}}{n} A^{\amalg^{n}} .
$$

In the case of a field $\mathbb{K}$ of positive characteristic $p$, the function $\log _{\text {! }}$ is again given by the restriction to $1+\mathfrak{m}$ of a $p$-homogeneous form $\mu_{p}$.

The form $\mu_{p}$ has all its coefficients in $\mathbb{N}$ and is again defined by the equality

$$
A^{\sqcup^{p}}=(A, 1)^{p}+p \mu_{p}(A)
$$

over $\mathbb{Z}$. It can thus be defined over an arbitrary field.

\section{Rational series}

We say that a formal power series $A$ is rational if it belongs to the smallest subalgebra in $\mathbb{K}\left\langle\left\langle X_{1}, \ldots, X_{k}\right\rangle\right\rangle$ that contains the free associative algebra $\mathbb{K}\left\langle X_{1}, \ldots, X_{k}\right\rangle$ of noncommutative polynomials and intersects the group of multiplicative units of $\mathbb{K}\left\langle\left\langle X_{1}, \ldots, X_{k}\right\rangle\right\rangle$ in a subgroup. 
Given a monomial $\boldsymbol{T} \in \mathscr{X}^{*}$, we denote by

$$
\rho(\boldsymbol{T}): \mathbb{K}\left\langle\left\langle X_{1}, \ldots, X_{k}\right\rangle\right\rangle \longrightarrow \mathbb{K}\left\langle\left\langle X_{1}, \ldots, X_{k}\right\rangle\right\rangle
$$

the linear application defined by

$$
\rho(\boldsymbol{T}) A=\sum_{\boldsymbol{X} \in \mathscr{L}^{*}}(A, \boldsymbol{X} \boldsymbol{T}) \boldsymbol{X}
$$

for $A=\sum_{\boldsymbol{X} \in \mathscr{L}}(A, \boldsymbol{X}) \boldsymbol{X}$ in $\mathbb{K}\left\langle\left\langle X_{1}, \ldots, X_{k}\right\rangle\right)$. The identity

$$
\rho(\boldsymbol{T})\left(\rho\left(\boldsymbol{T}^{\prime}\right) A\right)=\rho\left(\boldsymbol{T} \boldsymbol{T}^{\prime}\right) A
$$

shows that we have a representation

$$
\rho: \mathscr{X}^{*} \longrightarrow \operatorname{End}(\mathbb{K}\langle\langle\mathscr{X}\rangle)
$$

of the free monoid $\mathscr{L}^{*}$ on $\mathscr{X}$. The recursive closure $\bar{A}$ of a power series $A$ is the vector space spanned by its orbit $\rho\left(\mathscr{Q}^{*}\right) A$ under $\rho\left(\mathscr{C}^{*}\right)$. We call the dimension $\operatorname{dim} \bar{A}$ of $\bar{A}$ the complexity of $A$.

We call a subspace $\mathscr{A} \subset \mathbb{K}\left\langle\left\langle X_{1}, \ldots, X_{k}\right\rangle\right.$ recursively closed if it contains the recursive closure of all its elements.

Rational series coincide with series of finite complexity by a theorem of Schützenberger [Berstel and Reutenauer 1988, Theorem 1].

Remark 9.1. In the case of one variable, the complexity $\operatorname{dim} \bar{A}$ of a reduced nonzero rational fraction $A=f / g$ with $f \in \mathbb{K}[X]$ and $g \in 1+X \mathbb{K}[X]$ equals $\operatorname{dim} \bar{A}=\max (1+\operatorname{deg} f, \operatorname{deg} g)$.

Remark 9.2. The (generalized) Hankel matrix $H=H(A)$ of

$$
A=\sum_{\boldsymbol{X} \in \mathscr{Q}^{*}}(A, \boldsymbol{X}) \boldsymbol{X} \in \mathbb{K}\left\langle\left\langle X_{1}, \ldots, X_{k}\right\rangle\right.
$$

is the infinite matrix with rows and columns indexed by the free monoid $\mathscr{X}^{*}$ of monomials and entries $H_{\boldsymbol{X} \boldsymbol{X}^{\prime}}=\left(A, \boldsymbol{X} \boldsymbol{X}^{\prime}\right)$. The rank of $H$ is given by the complexity $\operatorname{dim} \bar{A}$ of $A$, and $\bar{A}$ corresponds to the column-span of $H$.

Given subspaces $\mathscr{A}, \mathscr{B}$ of $\mathbb{K}\langle\langle\mathscr{Q}\rangle\rangle$, we denote by $\mathscr{A} \amalg \mathscr{B}$ the vector space spanned by all products $A \amalg B$ with $A \in \mathscr{A}$ and $B \in \mathscr{B}$.

Proposition 9.3. We have the inclusion $\overline{A \amalg B} \subset \bar{A} \amalg \bar{B}$ for the shuffle product $A \amalg B$ of $A, B \in \mathbb{K}\left\langle\left\langle X_{1}, \ldots, X_{k}\right\rangle\right\rangle$.

Corollary 9.4 [Fliess 1974, Proposition 4]. We have

$$
\operatorname{dim}(\overline{A \amalg B}) \leq \operatorname{dim} \bar{A} \operatorname{dim} \bar{B}
$$

for the shuffle product $A \amalg B$ of $A, B \in \mathbb{K}\left\langle\left\langle X_{1}, \ldots, X_{k}\right\rangle\right\rangle$. In particular, shuffle products of rational elements in $\mathbb{K}\left\langle\left\langle X_{1}, \ldots, X_{k}\right\rangle\right\rangle$ are rational. 
Proof of Proposition 9.3. For $Y \in \bar{A}, Z \in \bar{B}$ and $X$ in $\left\{X_{1}, \ldots, X_{k}\right\}$, the recursive definition of the shuffle product given in Section 8 shows

$$
\rho(X)(Y \amalg Z)=(\rho(X) Y) \sqcup Z+Y \amalg(\rho(X) Z) .
$$

We thus have the inclusions $\rho(X)(Y \sqcup Z) \in \bar{A} \sqcup Z+Y \amalg \bar{B} \subset \bar{A} \amalg \bar{B}$, which show that the vector space $\bar{A} \amalg \bar{B}$ is recursively closed. Proposition 9.3 follows now from the inclusion $A \sqcup B \in \bar{A} \sqcup \bar{B}$.

Remark 9.5. Similar arguments show that the set of rational elements in $\mathbb{K} \ll\left\langle X_{1}\right.$, $\left.\ldots, X_{k}\right\rangle$ is also closed under the ordinary product (and multiplicative inversion of invertible series), Hadamard product and composition (where one considers $A \circ$ $\left(B_{1}, \ldots, B_{k}\right)$ with $A \in \mathbb{K}\left\langle\left\langle X_{1}, \ldots, X_{k}\right\rangle\right\rangle$ and $\left.B_{1}, \ldots, B_{k} \in \mathfrak{m} \subset \mathbb{K}\left\langle\left\langle X_{1}, \ldots, X_{k}\right\rangle\right\rangle\right)$.

Remark 9.6. The shuffle inverse of a rational element in $\mathbb{K}^{*}+\mathfrak{m}$ is in general not rational in characteristic 0 . An exception is given by geometric progressions

$$
\left(1-\sum_{j=1}^{k} \lambda_{j} X_{j}\right)^{-1}=\sum_{n=0}^{\infty}\left(\sum_{j=1}^{k} \lambda_{j} X_{j}\right)^{n}
$$

since we have

$$
\frac{1}{1-\sum_{j=1}^{k} \lambda_{j} X_{j}} \amalg \frac{1}{1-\sum_{j=1}^{k} \mu_{j} X_{j}}=\frac{1}{1-\sum_{j=1}^{k}\left(\lambda_{j}+\mu_{j}\right) X_{j}}
$$

corresponding to $e^{\lambda X} e^{\mu X}=e^{(\lambda+\mu) X}$ in the one-variable case.

There are no other such elements in $1+\mathfrak{m} \subset \mathbb{K} \llbracket X \rrbracket$; see Remark 4.2. I do not know whether the maximal rational shuffle subgroup of $1+\mathfrak{m} \subset \mathbb{C}\left\langle\left\langle X_{1}, \ldots, X_{k}\right\rangle\right.$ (defined as the set of all rational elements in $1+\mathfrak{m}$ with rational inverse for the shuffle product) contains other elements if $k \geq 2$.

Remark 9.7. Any finite set of rational elements in $\mathbb{K}\left\langle\left\langle X_{1}, \ldots, X_{k}\right\rangle\right\rangle$ over a field $\mathbb{K}$ of positive characteristic is included in a unique minimal finite-dimensional recursively closed subspace of $\mathbb{K}\left\langle\left\langle X_{1}, \ldots, X_{k}\right\rangle\right\rangle$ that intersects the shuffle group $\left(\mathbb{K}^{*}+\mathfrak{m}, \amalg\right)$ in a subgroup.

\section{Main result for generating series in noncommuting variables}

The following statement is our main result in a noncommutative framework.

Theorem 10.1. Let $\mathbb{K}$ be a subfield of $\overline{\mathbb{F}}_{p}$. Given a noncommutative formal power series $A \in \mathfrak{m} \subset \mathbb{K}\langle\langle\mathscr{X}\rangle\rangle$, the following two assertions are equivalent:

- A is rational.

- $\exp _{\text {! }}$ A is rational. 
More precisely, we have for a rational series $A$ in $\mathfrak{m}$ the inequalities

$$
\operatorname{dim} \overline{\log _{!}(1+A)} \leq 1+(\operatorname{dim} \overline{1+A})^{p}
$$

and

$$
\operatorname{dim} \overline{\exp ! A} \leq p^{q^{\operatorname{dim} \bar{A}}}
$$

where $q=p^{e}$ is the cardinality of a finite field $\mathbb{F}_{q}$ containing all coefficients of $A$. Proof. The identity

$$
\log _{!}(1+A)=\sum_{X \in \mathscr{X}}\left((1+A) \uplus^{p-1} \amalg \rho(X) A\right) X
$$

and Corollary 9.4 show

$$
\operatorname{dim} \overline{\log _{!}(1+A)} \leq 1+(\operatorname{dim} \overline{1+A})^{p} .
$$

For the opposite direction we denote by $\mathbb{K}=\mathbb{F}_{q}$ a finite subfield of $\overline{\mathbb{F}}_{p}$ containing all coefficients of $A$. We have

$$
\overline{\exp _{!} A} \subset \exp ! A \sqcup \mathbb{K}[\Gamma(A)],
$$

where $\mathbb{K}[\Gamma(A)]$ is the shuffle subalgebra of dimension at most $p^{q^{\operatorname{dim} \bar{A}}}$ spanned by all elements of the group $\Gamma$ generated by all elements of the form

$$
(\bar{A}+\mathbb{K}) \cap(1+\mathfrak{m}) .
$$

This implies the inequality $\operatorname{dim} \overline{\exp ! A} \leq p^{q^{\operatorname{dim} \bar{A}}}$, which ends the proof.

\section{Acknowledgements}

I thank Jean-Paul Allouche, Michel Brion, Alexei Panchishkin, Tanguy Rivoal, Boris Venkov and an anonymous referee for their interest and helpful remarks.

\section{References}

[Allouche and Shallit 2003] J.-P. Allouche and J. Shallit, Automatic sequences, Cambridge University Press, 2003. MR 2004k:11028 Zbl 1086.11015

[Bacher 2006] R. Bacher, "La suite de Thue-Morse et la catégorie Rec", C. R. Math. Acad. Sci. Paris 342:3 (2006), 161-164. MR 2006g:11050 Zbl 1148.11012

[Bacher 2008] R. Bacher, "Determinants related to Dirichlet characters modulo 2, 4 and 8 of binomial coefficients and the algebra of recurrence matrices", Internat. J. Algebra Comput. 18:3 (2008), 535-566. MR 2009c:15006 Zbl 1190.15005

[Berstel and Reutenauer 1988] J. Berstel and C. Reutenauer, Rational series and their languages, EATCS Monographs on Theoretical Computer Science 12, Springer, Berlin, 1988. Revised edition available at http://tagh.de/tom/wp-content/uploads/berstelreutenauer2008.pdf. MR 90e:68054 Zbl 0668.68005

[Berthelot and Ogus 1978] P. Berthelot and A. Ogus, Notes on crystalline cohomology, Princeton University Press, 1978. MR 58 \#10908 Zbl 0383.14010 
[Cartier 2002] P. Cartier, "Fonctions polylogarithmes, nombres polyzêtas et groupes pro-unipotents", pp. 137-173 in Séminaire Bourbaki 2000/2001 (Exposé 885), Astérisque 282, Soc. Mat. de France, Paris, 2002. MR 2004i:19005

[Christol 1979] G. Christol, "Ensembles presque periodiques $k$-reconnaissables", Theoret. Comput. Sci. 9:1 (1979), 141-145. MR 80e:68141 Zbl 0402.68044

[Comtet 1970] L. Comtet, Analyse combinatoire, II, Le Mathématicien 5, Presses Universitaires de France, Paris, 1970. MR 41 \#6697 Zbl 0221.05002

[Fliess 1974] M. Fliess, "Sur divers produits de séries formelles", Bull. Soc. Math. France 102 (1974), 181-191. MR 50 \#7125 Zbl 0313.13021

[Hurwitz 1899] A. Hurwitz, "Über die Entwickelungscoefficienten der lemniskatischen Functionen”, Math. Ann. 51 (1899), 196-226. JFM 29.0385.02

[Lucas 1878] E. Lucas, "Sur les congruences des nombres eulériens et les coefficients différentiels des fonctions trigonométriques suivant un module premier", Bull. Soc. Math. France 6 (1878), 4954. MR 1503769 JFM 10.0139.04

[Nekrashevych 2005] V. Nekrashevych, Self-similar groups, Mathematical Surveys and Monographs 117, American Mathematical Society, Providence, RI, 2005. MR 2006e:20047 Zbl 1087.20032

[Reutenauer 1993] C. Reutenauer, Free Lie algebras, London Mathematical Society Monographs. New Series 7, Oxford University Press, 1993. MR 94j:17002 Zbl 0798.17001

[Roby 1963] N. Roby, "Lois polynomes et lois formelles en théorie des modules", Ann. Sci. École Norm. Sup. (3) 80 (1963), 213-348. MR 28 \#5091 Zbl 0117.02302

[Roby 1965] N. Roby, "Les algèbres à puissances divisées", Bull. Sci. Math. (2) 89 (1965), 75-91. MR 33 \#1348 Zbl 0145.04503

[Stanley 1999] R. P. Stanley, Enumerative combinatorics, II, Cambridge Studies in Advanced Mathematics 62, Cambridge University Press, 1999. MR 2000k:05026 Zbl 0928.05001

[Zagier 1994] D. Zagier, "Values of zeta functions and their applications", pp. 497-512 in First European Congress of Mathematics (Paris, 1992), vol. 2, edited by A. Joseph et al., Progr. Math. 120, Birkhäuser, Basel, 1994. MR 96k:11110 Zbl 0822.11001

Communicated by Hendrik W. Lenstra

Received 2009-08-24 Revised 2010-07-13 Accepted 2010-10-17

Roland.Bacher@ujf-grenoble.fr Université Grenoble I, CNRS UMR 5582, Institut Fourier, 100, rue des Maths, Boîte Postale 74, 38402 St. Martin d'Hères, France 


\section{Algebra \& Number Theory}

www.jant.org

\section{EDITORS}

\section{MANAGING EDITOR}

Bjorn Poonen

Massachusetts Institute of Technology

Cambridge, USA

\author{
EDITORIAL BOARD CHAIR \\ David Eisenbud \\ University of California \\ Berkeley, USA
}

\section{BOARD OF EDITORS}

\section{Georgia Benkart}

Dave Benson

Richard E. Borcherds

John H. Coates

J-L. Colliot-Thélène

Brian D. Conrad

Hélène Esnault

Hubert Flenner

Edward Frenkel

Andrew Granville

Joseph Gubeladze

Ehud Hrushovski

Craig Huneke

Mikhail Kapranov

Yujiro Kawamata

János Kollár

Hendrik W. Lenstra

Yuri Manin

Barry Mazur
University of Wisconsin, Madison, USA

University of Aberdeen, Scotland

University of California, Berkeley, USA

University of Cambridge, UK

CNRS, Université Paris-Sud, France

University of Michigan, USA

Universität Duisburg-Essen, Germany

Ruhr-Universität, Germany

University of California, Berkeley, USA

Université de Montréal, Canada

San Francisco State University, USA

Hebrew University, Israel

University of Kansas, USA

Yale University, USA

University of Tokyo, Japan

Princeton University, USA

Universiteit Leiden, The Netherlands

Northwestern University, USA

Harvard University, USA
Susan Montgomery

Shigefumi Mori

Andrei Okounkov

Raman Parimala

Victor Reiner

Karl Rubin

Peter Sarnak

Michael Singer

Ronald Solomon

Vasudevan Srinivas

J. Toby Stafford

Bernd Sturmfels

Richard Taylor

Ravi Vakil

Michel van den Bergh

Marie-France Vignéras

Kei-Ichi Watanabe

Andrei Zelevinsky

Efim Zelmanov
University of Southern California, USA

RIMS, Kyoto University, Japan

Princeton University, USA

Emory University, USA

University of Minnesota, USA

University of California, Irvine, USA

Princeton University, USA

North Carolina State University, USA

Ohio State University, USA

Tata Inst. of Fund. Research, India

University of Michigan, USA

University of California, Berkeley, USA

Harvard University, USA

Stanford University, USA

Hasselt University, Belgium

Université Paris VII, France

Nihon University, Japan

Northeastern University, USA

University of California, San Diego, USA

\section{PRODUCTION}

ant@mathscipub.org

Silvio Levy, Scientific Editor

Andrew Levy, Production Editor

See inside back cover or www.jant.org for submission instructions.

The subscription price for 2010 is US \$140/year for the electronic version, and \$200/year (+\$30 shipping outside the US) for print and electronic. Subscriptions, requests for back issues from the last three years and changes of subscribers address should be sent to Mathematical Sciences Publishers, Department of Mathematics, University of California, Berkeley, CA 94720-3840, USA.

Algebra \& Number Theory (ISSN 1937-0652) at Mathematical Sciences Publishers, Department of Mathematics, University of California, Berkeley, CA 94720-3840 is published continuously online. Periodical rate postage paid at Berkeley, CA 94704, and additional mailing offices.

ANT peer review and production are managed by EditFLOW ${ }^{\mathrm{TM}}$ from Mathematical Sciences Publishers.

\section{PUBLISHED BY}

mathematical sciences publishers

http://www.mathscipub.org

A NON-PROFIT CORPORATION

Typeset in LATEX

Copyright $\odot 2010$ by Mathematical Sciences Publishers 


\section{Algebra \& Number Theory}

Volume $4 \quad$ No. $7 \quad 2010$

Hochschild cohomology and homology of quantum complete intersections

STEFFEN OPPERMANN

Meromorphic continuation for the zeta function of a Dwork hypersurface

THOMAS BARNET-LAMB

Equations for Chow and Hilbert quotients

ANGEla Gibney and Diane MaClagan

Haglund-Haiman-Loehr type formulas for Hall-Littlewood polynomials of type $B$ and C

\section{CRISTIAN LENART}

On exponentials of exponential generating series

ROLAND BACHER

On families of $\varphi, \Gamma$-modules

KIRAN KEDLAYA and RUOCHUAN LIU 